\title{
Design of AI Meal-Assisting Manipulator via Open-Mouth Target Detection
}

\author{
Zhongliang Yang ${ }^{\mathrm{a}, \mathrm{b}, 1}$, Xingli Jia ${ }^{\mathrm{b}}$,Yifan Dai ${ }^{\mathrm{b}}$,Xinyu Zhang ${ }^{\mathrm{b}}$, and Jialu Tang ${ }^{\mathrm{b}}$ \\ ${ }^{a}$ Design Intelligence Innovation Center, China Academy of Art, Hangzhou 310024, \\ China \\ yzl@dhu.edu.cn \\ ${ }^{\mathrm{b}}$ College of Mechanical Engineering, Donghua University, Shanghai 201620, China
}

\begin{abstract}
To help the disabled people eat, this paper proposed a AI meal-assisting manipulator via the detection of the open-mouth target. The YOLO_V3 algorithm was used to complete the construction of the mouth opening and closing state data set and model training, and the mouth state recognition model was successfully constructed and evaluated. The experimental results show that the model has a high recognition accuracy rate, accuracy rate and recall rate of the mouth opening and closing state. Finally, the application verification was successfully achieved by building a functional prototype of the meal-assisting manipulator.
\end{abstract}

Keywords. Target detection, meal-assisting manipulator, YOLO_V3, Artificial Intelligence

\section{Introduction}

Disabled people refer to people who have lost the ability to take care of themselves and need caregivers to take care of their diet and daily life. However, as the aging of the population accelerates and the number of disabled people continues to increase, the number of existing care workers can no longer meet the needs of disabled people. In food, clothing, housing and transportation, "food" is the most basic need of the disabled. Therefore, it is of great research significance to use meal-assisting machinery and equipment to improve human resources. The interaction mode of the existing mealassisting robots has certain restrictions on the user population, the use environment, etc., and there is often a problem of disconnection between the design concept and the technical realization. Therefore, it is very necessary to broaden the interaction methods of the existing intelligent food-assisting robots and complete the application verification of the intelligent food-assisting robots.

From the perspective of the development of food-assisting robots, the research of food-assisting robots originated in Europe and the United States ${ }^{[1]}$. In the 1980s, the manipulators RSI and MANUS service robots developed by the Netherlands and France ${ }^{[2][3]}$ promoted the development of subsequent meal-assisting service robots.

\footnotetext{
${ }^{1}$ Corresponding Author
} 
In 1987, the British company Mike Topping developed a food aid robot Handy1 to help an 11-year-old boy with cerebral palsy complete basic nursing activities such as eating ${ }^{[4][5]}$, as shown in Figure 1. In 1989, more integrated and modular Handy1 type II was developed, which has replaceable tray components to meet the needs of patients in eating, washing, makeup, leisure and entertainment ${ }^{[4][5][6]}$. Handy1 is considered to be one of the most successful rehabilitation robots in the world, but because Handy1 integrates a variety of functions related to the diet and daily life of disabled people, the robot is relatively cumbersome, which makes Handy1 not widely applicable ${ }^{[7]}$.

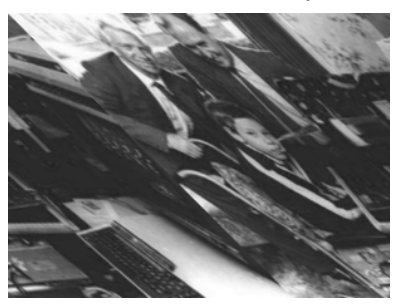

Figure 1. Handy1.

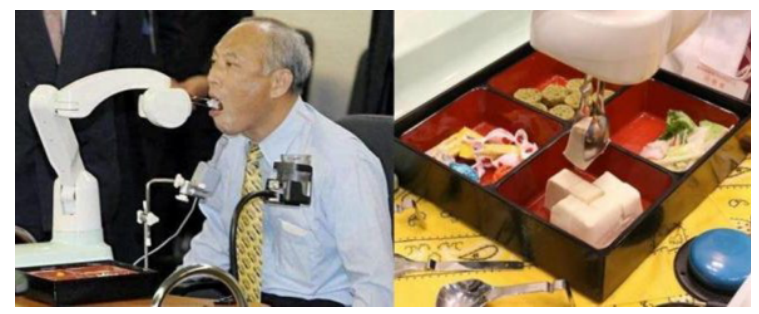

Figure 2. My SPOON feeding robot.

In 2001, the Japanese company SECOM announced the 11-year-old robot "MY SPOON" at the 28th International Welfare Equipment Exhibition to help people with disabilities or injuries caused by inconvenience to recognize and eat ${ }^{[7][8]}$, as shown in Figure 2. MY SPOON has a 6-degree-of-freedom robotic arm, and the way of taking food is gripping. It has the characteristics of compact structure, small size and strong flexibility. In addition, people with disabilities can also choose the control mode according to their disability.

From the perspective of the development of food-assisting robots at home and abroad, food-assisting robots are service-oriented robots. Compared with foreign countries, domestic research on meal-assisting robots started late, with fewer achievements, and no mature products. The appearance, interaction method, and mechanical control are not well integrated, and there is a disconnect between the design and implementation layers Phenomenon.

From the perspective of interaction and control methods, the existing food assistance robots can be roughly divided into three types: one is the control method of pedal and finger switches or joysticks, and the user controls the food assistance robot to feed by pressing or stepping; the second type is voice recognition control. The voice signal of the specified function is stored in the template library. When the user issues a voice command, it is matched and recognized with the existing signal in the template library, and then the relevant instructions are executed; the third type is the recognition head Partial posture drives the meal-assisting robot to assist with meals, such as pre-setting head shaking, head-down and other actions corresponding to the specific needs of the user, and generating corresponding operation commands by recognizing the user's action 
state during use ${ }^{[9]}$. Among the three control methods, the foot switch and finger button control are more direct and easy to implement, but they have requirements and restrictions on the user's physical activity. The voice recognition control is greatly affected by the environment, and it is incompatible with the Chinese nation. The habit of "sleeping silently" is not consistent; heading recognition is the least demanding for users, but in terms of user behavior, the additional head gestures such as bowing and shaking their heads are not connected smoothly with the eating behavior. Analyzed from the behavioral habits of humans, animals, etc., "opening the mouth" is a natural behavior before every meal, which is more in line with human behavioral habits. Therefore, if the mouth opening and closing state can be tested, the user's "mouth opening" characteristics can be used to drive. The meal-assisting robot will further complement and innovate the interaction methods and control strategies of the existing meal-assisting robots.

From the point of view of the method suitable for mouth opening and closing state detection, the model needs to classify and locate the image at the same time-find the image of the opened mouth and locate the position of the mouth for delivery. Therefore, the target detection technology based on deep learning is the most. It is suitable for building a mouth state recognition model. Among them, the mouth opening and closing state belongs to two categories, and the characteristics are more obvious. The model built by the YOLO_V3 algorithm will have a better performance in the balance of accuracy and speed.

Based on the above research status, this article mainly focuses on the following two issues:

Broaden the interaction methods of existing meal-assisting robots. Therefore, it is necessary to expand and innovate the existing human-computer interaction methods. This paper proposes a new interactive mode of "feeding by opening the mouth to drive the meal-assisting manipulator", which has lower requirements for the user's activity ability and conforms to the behavior logic of people opening their mouths before eating. The difficulty lies in the establishment of the mouth opening and closing state classification data set, and the establishment of a mouth state recognition model through computer vision related algorithms.

Complete the application verification of the intelligent meal-assisting manipulator. The existing domestic intelligent food-assisting robots mostly stay in the research stage, and there is a phenomenon that the product design stage and the design realization stage are out of touch. This article hopes to integrate knowledge related to industrial design, mechanical structure, electromechanical engineering, computer vision, and intelligent hardware through knowledge integration to complete the application verification of the intelligent meal-assisting manipulator.

\section{Methods}

\subsection{Mouth state recognition model structure via YOLO_V3}

The structure of the mouth state recognition model can be divided into three modules: feature extraction module, feature processing module, mouth recognition and positioning module. In the mouth state recognition model, the structure of Darknet-53 is used as the mouth state feature extraction module. The green image in Figure 3 is the structure of the feature extraction module. When a Padding mouth state image is input into the feature extraction module, it must be convolved once, and the number of channels is increased 
to 32 ; then through five residual networks, the final feature dimension is $13 * 13$, and the number of channels is 1024 feature image. Compared with ordinary convolution, the residual network can directly map the previous feature layer to the latter feature layer, which can better perform feature extraction and improve the accuracy. It can also alleviate the problem of gradient disappearance as the neural network deepens ${ }^{[10][11]}$. The five residual networks are all composed of a single convolutional layer and a set of repeated convolutional layers. The repeated convolution units of the five residual networks are repeated $1,2,8,8$, and 4 times respectively. In each residual network, since the convolution step length performed separately is 2 , the size of the feature image becomes one-half of the original after each layer of residual network. In the end, the feature extraction network of Darknet-53 will retain the last three feature images of size $52 * 52,26 * 26,13 * 13$, and input them into the feature processing network of YOLO_V3 to wait for the next step of processing.

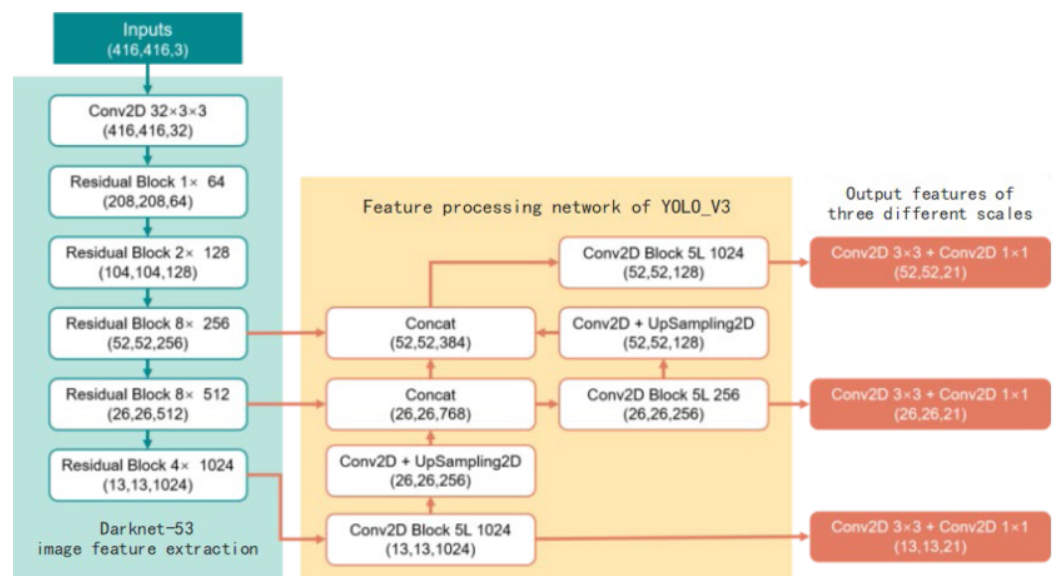

Figure 3. Network structure of mouth state recognition model.

The mouth state recognition model adopts the feature processing network structure of YOLO_V3 as the mouth state feature processing module. As shown in the yellow image part in Figure 3, first, the $13 * 13$ feature image with 1024 channels obtained in Darknet-53 is convolved five times, and the feature image after convolution has two destinations: First, directly output the final feature with a size of $13^{*} 13$ and a channel number of 21; the second direction is that after upsampling, the length and width are expanded to twice the original feature, and the $26 * 26$ feature output by Darknet-53 Layers are stacked. Then, the stacked feature layer is convolved five times to obtain the second feature with a size of $26^{*} 26$ and a channel number of 21 . Finally, the $26 * 26$ feature layer is up-sampled and stacked to obtain the final feature image with a size of $52 * 52$ and a channel number of 21 . As can be seen from the orange part of Figure 3, YOLO_V3 outputs a total of three different scale feature layers, representing the prediction results of three different scales. Among them, 13, 26, 52 represent the degree of grid division, which is suitable for the detection of large, medium and small objects respectively; 21 represents 21 channels, which can be understood and divided into $3 \times$ $(4+1+2)$ channels-3 represents Three a priori boxes, 4 represents the four data of each a priori box, namely the width $\mathrm{w}$ of the rectangular box where the mouth is, the height $\mathrm{h}$, the coordinates of the center point of the rectangular box (Xp, Yp), and 1 represents this category The confidence level of 2 represents the number of categories, namely "open 
mouth" and "close mouth", that is, 21 channels record the characteristics of the input picture in detail.

The mouth recognition and positioning module is actually a process of decoding the three-scale feature layers obtained by the YOLO_V3 feature processing network. First, use a priori box to judge the characteristics of each small grid. There are nine a priori boxes, three in each group, corresponding to three kinds of grids with different division degrees, of which the $13 * 13$ grid corresponds to the largest three A priori box, the $52 * 52$ grid corresponds to the smallest three a priori boxes; then, the small grid with "open mouth" feature is similarly added and averaged, and finally the entire "open mouth" image is obtained. Location (indicated by the offset of the center point $\mathrm{x}$ and $\mathrm{y}$, the length and width of the rectangular box w, h), confidence, and the possibility of the category, compare the confidence of the three scale feature images, and choose the preferred one; Since it corresponds to the offset of the Padding $416^{*} 416$ picture (that is, the image with gray borders) at this time, it should be converted into the offset of the center store corresponding to the actual picture; finally, the center point of the rectangular box corresponds to the actual The offset $\mathrm{x}$ _offset, $\mathrm{y} \_$offset of the picture and the length and width $\mathrm{w}, \mathrm{h}$ of the rectangular frame are calculated, and the coordinates of the points at the upper left corner $\mathrm{M}$ and the lower right corner $\mathrm{N}$ of the rectangular frame are calculated, and the position of the mouth is framed in the actual picture by using the coordinates. After the above decoding process, the mouth state recognition model shows us the type of mouth closed picture it judges and the position of the mouth in the language of the illustration.

\subsection{Image acquisition and classification standards}

The opening and closing state of the mouth is generally divided into two states: "mouth open" and "mouth closed" (open and closed will be used later for a brief description). However, in practical applications, specific situations still need to be classified in detail. This article will use the controlled variable method to analyze the image collection and classification standards.

(1) Collection of different mouth states under the same background

Collect multiple states of mouth opening and closing under the same background. Among them, "on" takes the width of one finger of the person being photographed and the maximum opening angle of the upper and lower jaws of the person being photographed as the two limit ranges of the "on" state of the captured picture. And within this range, collect multiple pictures. "Close" is mainly divided into the following three types: first, the mouth is fully closed; second, the mouth opening range is less than the width of the subject's finger and it is judged as closed, which corresponds to the user's mouth during chewing in the actual state Unconsciously slightly open; thirdly, the lips are separated but the teeth are closed and judged to be closed.

(2) Different backgrounds in the same state

Taking into account the different dining scenes, when collecting pictures, it is necessary to collect pictures with diverse backgrounds as much as possible when the mouth opening and closing state are the same, so as to improve the robustness of the model. Increase the accuracy of training results, as shown in Figure 4. 


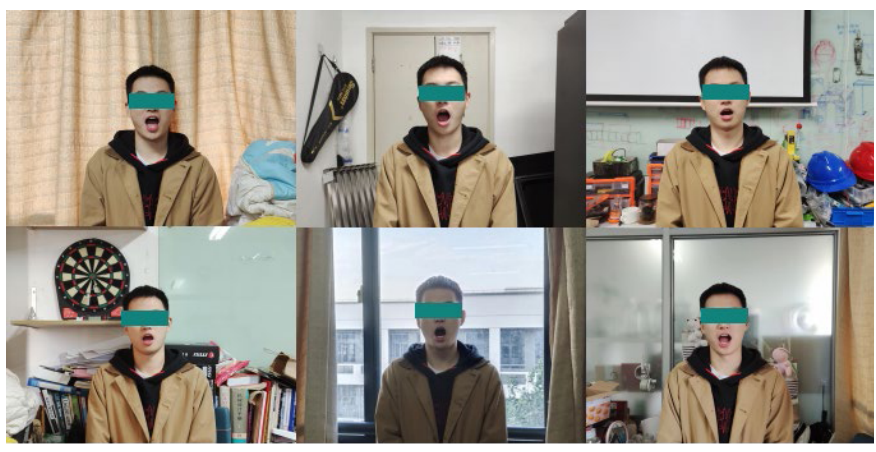

Figure 4. Collection of different backgrounds with mouth open.

\subsection{Process of image collection and image annotation}

The acquisition process was carried out according to the image acquisition standard in chapter 2.2, and a total of 113 mouth state images were collected, a total of 1040 images. Among them, the population covers ages ranging from 12 to 72 years old, and men and women are evenly distributed. The environmental background of the collected persons is diverse.

\subsection{Model training method}

Tool preparation; Select the operating environment; Install relevant frameworks and required libraries; Manual establishment of relevant folders, for the subsequent code to run the corresponding location; Import images and data set information; Make to main.py; Run the Python program voc annotation.py to record the type and location information of data sets divided into different parts. Change the classes and convolution core filters parameters in the configuration file. Convert Darknet-based YOLO to H5 files available for Keras; Modify the classification of model-related data; Clustering method to generate feature selection box; Modify the path information and some data in the training file to prepare for training; Modify hyperparameters; Run train.py and wait for the training to complete.

\subsection{Correspondence between mouth state recognition and delivery position}

This section will focus on the corresponding method of "mouth position" and "food delivery position" in the open mouth state.

Step1: Construct the two-dimensional coordinate system of the picture taken by the Raspberry Pi camera.

The picture taken by the Raspberry Pi camera is a two-dimensional plane with a fixed range. With the upper left corner as the origin, a coordinate system as shown in Figure 5 can be established. Each pixel is a unit. The maximum coordinate $M$ (that is, the coordinate of the point at the lower right corner of the picture) is $(1280,800)$. After the mouth position is recognized by the mouth state recognition model, the coordinate point of the rectangular frame where the mouth is located will be output. That is, in Figure 5, the coordinate position of the upper left corner point $\mathrm{P}$ and the lower right corner point $\mathrm{Q}$ of the rectangular box in this coordinate system. 


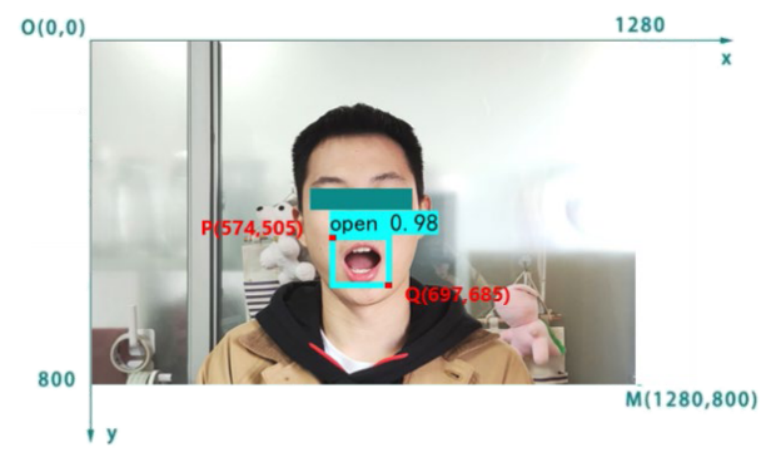

Figure 5. The corresponding coordinate system of the picture taken by the Raspberry Pi camera.

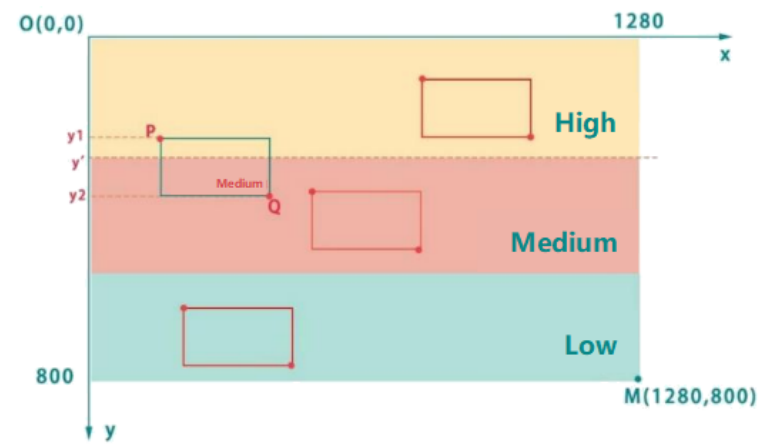

Figure 6. Correspondence between the delivery range and the coordinate system of the taken picture.

Step2: Divide the area of the food delivery location.

In the sitting state, the sitting height will be different according to the height of the user. And when the user is in a sitting state, the height is difficult to greatly change, and the body has a certain degree of mobility and freedom in the front and rear, left and right positions. Therefore, this study divided the three food delivery areas of "high, medium, and low" according to the height of different groups of people, and used these three positions as a simplified model to verify the feasibility of the plan.

Step3: Clarify the correspondence between the two-dimensional coordinate system of the picture taken by the Raspberry Pi camera and the delivery location.

The two-dimensional coordinate system captured by the camera corresponds to the location plane of the food delivery area, and the coordinate system is also divided into three ranges of high, medium, and low, as shown in Figure 6. The small rectangular frame in the figure is the mouth position of the mouth picture recognized by the mouth state recognition model. The coordinates of the upper left and lower right corners of the rectangular marquee are compared with the y-axis boundary coordinates of the three regions of high, medium, and low. You can get the corresponding delivery location. For the special case of the green selection box in the figure, the solution is as follows, then the $y$-axis coordinates $\mathrm{y} 1$ and $\mathrm{y} 2$ of point $\mathrm{P}$ and point $\mathrm{Q}$ are calculated with the $\mathrm{y}$-axis coordinate y'of the boundary line of the middle and high area respectively, and the difference is compared The absolute value of the operation is to compare the size of $\left|y 1-y^{\prime}\right|$ and $\left|y 2-y^{\prime}\right|$ in the figure. Finally, the food delivery location is classified as the area where the point with the greater absolute value of the difference calculation is located. Take the green selection box in the figure as an example, $\left|y 1-y^{\prime}\right|<\left|y 2-y^{\prime}\right|$, it 
is divided according to the area where point $\mathrm{Q}$ is located, and the robotic arm will deliver the food to the middle position.

\section{Results and discussion}

\subsection{The results of the mouth state recognition model}

The current model has a good convergence effect, and the recognition accuracy has reached 93.45\%, as shown in Figure 7.

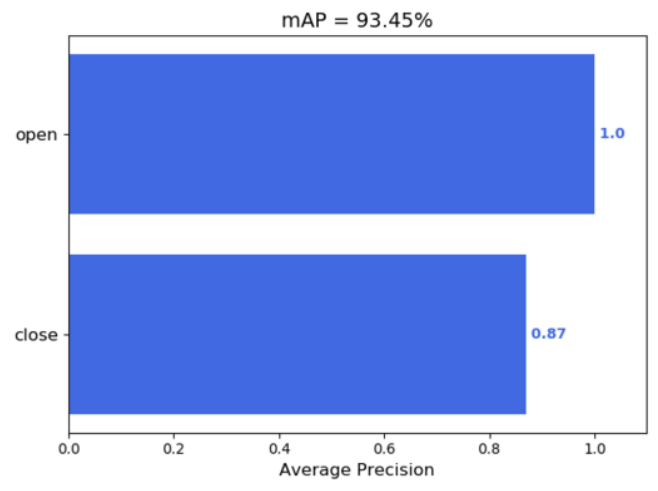

Figure 7. Model recognition accuracy.

\subsection{Evaluation of Mouth State Recognition Model}

In this paper, two indicators of recall and precision in the process of model testing are used to evaluate the performance of the mouth state recognition model ${ }^{[12]}$.

The calculation of precision and recall requires the use of four evaluation indicators, TP, TN, FP, and FN. The four values represent the relationship between the true value of the positive and negative samples and the predicted value. Taking "open mouth" as the identification target, $\mathrm{T}$ and $\mathrm{F}$ indicate whether the prediction result is correct or wrong, $\mathrm{P}$ indicates that the prediction result is open mouth, and $\mathrm{N}$ indicates that the prediction result is other. The corresponding meaning of the two-letter combination is as follows:

TP: Actually "open mouth", which is predicted as "open mouth" by the mouth state recognition model;

TN: Actually "shut up or no mouth appears", which is predicted as "shut up or not recognized" by the mouth state recognition model;

FP: Actually "shut up or no mouth appears", predicted as "open mouth" by the mouth state recognition model;

FN: Actually "open mouth", predicted as "closed mouth or not recognized" by the mouth state recognition model.

The evaluation process uses 200 pictures as the test set used in this evaluation. The test results and corresponding accuracy and recall rates are shown in Table 1. It can be seen from the table that the mouth state recognition model has an accuracy rate of $97.3 \%$ for identifying "open mouth" and a recall rate of $91.8 \%$. The model detection result is relatively ideal. 
Table 1. Test set evaluation

\begin{tabular}{cccccc}
\hline Data category & TP & FP & FN & P & R \\
\hline Number of pictures & 181 & 5 & 16 & $97.3 \%$ & $91.8 \%$ \\
\hline
\end{tabular}

\section{Application verification}

A prototype of the machine was developed, as shown in Figure 8. Application verification is to connect each process of the above step-by-step verification to ensure the complete and smooth delivery process of the intelligent meal assistance manipulator. At the software level, it is necessary to transfer the recognition results of the mouth state recognition model as a parameter to the robotic arm motion program, so that the entire process is combined into a main program. The entire process of application verification is shown in Figure 9.

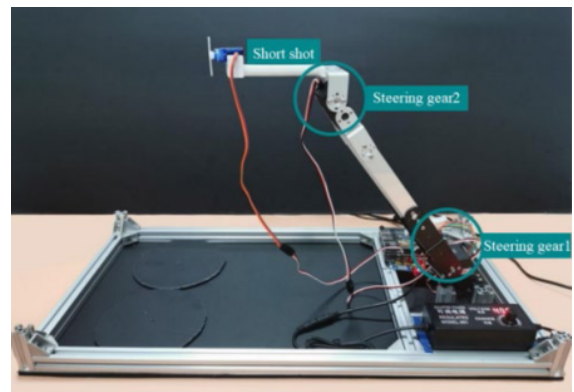

Figure 8. Machine prototype.

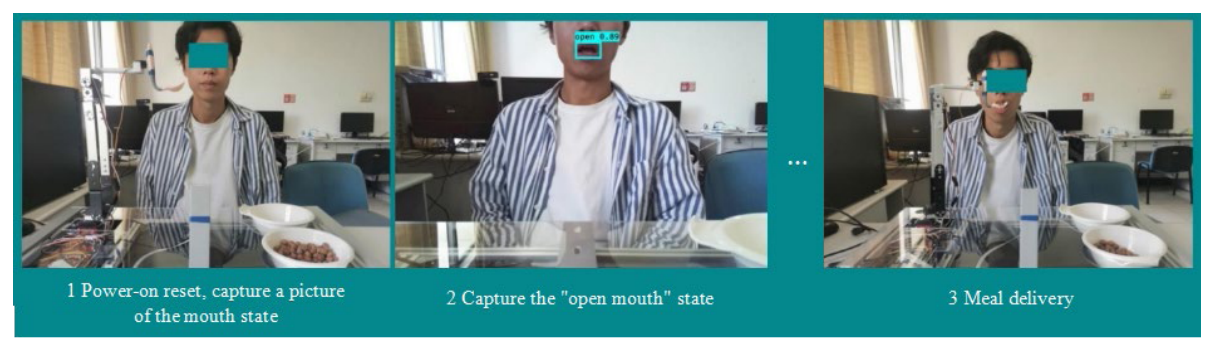

Figure 9. Application verification process.

First of all, after powering on, the robotic arm is in the reset state and does not block the face; the camera takes a picture in one second to capture the state of the mouth; the mouth state recognition model recognizes whether the taken picture is an open mouth picture; captures "open mouth" After taking the picture, the robotic arm starts to take the meal; after the meal is taken, the robotic arm is reset, and the current mouth is taken again to obtain the new mouth position (this step does not require the mouth to be in an open state, it is only for obtaining The current mouth position); finally, according to the mouth position, different food delivery positions are delivered, and the user eats. The above is the completion of a meal delivery process. After the meal delivery is completed, the robotic arm resets and prepares for the next meal delivery. During this application verification process, a total of 5 subjects participated in the test. During the experiment, the intelligent meal-assisting manipulator correctly recognized the testee's "mouth opening" signal and delivered the food to the correct area. It proves the reliability of the intelligent meal-assisting manipulator in the integrated design and development. 


\section{Conclusion}

First, the principle and structure of the established mouth state recognition model are analyzed; then the process of constructing the mouth opening and closing state data set is explained step by step, the image collection method and classification standard are clarified, and the image collection and labeling process is explained. Summarizes the relevant features of the mouth state opening and closing data set; then uses this data set for model training, and successfully establishes a mouth state recognition model; finally, evaluates and analyzes the results of the trained mouth state recognition model. The mouth state recognition model established in this paper has high accuracy, model accuracy and recall rate for the recognition of the mouth opening and closing state, and it has a very good recognition ability for the mouth opening and closing state of the face. Finally, through application verification, the mouth state recognition module, the intelligent meal-assisting manipulator control module and the intelligent meal-assisting manipulator hardware module are connected to complete all the functions of the intelligent meal-assisting manipulator, and the video of the use process is taken and recorded.

Acknowledgments. This study was partly supported by the Zhejiang Provincial Key Laboratory of Integration of Healthy Smart Kitchen System, China (No. 2014E10014) and the National Social Science Fund of Art, China (No. 20ZD09,18ZD19).

\section{References}

[1] Li Yantao. Research on the prototype and control of the meal-assisting robot[D]. Harbin: Harbin Engineering University, 2012.

[2] Gao Congjun. Development status and prospects of rehabilitation robots in Europe[J]. Robotics and Applications, 1999, 1999(05):2-4.

[3] Rosier J C, Woerden J, Kolk L, et al. Rehabilitation robotics: the MANUS concept[C]. Advanced Robotics, 1991.'Robots in Unstructured Environments', 91 ICAR. Fifth International Conference on. IEEE, 1991.

[4] Anonymous. The most successful rehabilitation robot in the world - Handy1[J]. Robotics and Applications, 2000(05):14-16.

[5] Topping M. An Overview of the Development of Handy 1, a Rehabilitation Robot to Assist the Severely Disabled[J]. Artificial Life \& Robotics, 2000, 4(4):188-192.

[6] TOPPING M J, SMITH J K. The development of handy 1. A robotic system to assist the severely disabled[J]. Technology and Disability, 1999, 10(2): 95-105.

[7] Yu Dafa. Research on the structure design and motion planning of Adroit food-assisting robot[D]. Harbin: Harbin Engineering University, 2013.

[8] Anonymous. SECOM showcases an auxiliary dining robot[J]. Robotics and Application, 2002(01):12.

[9] Wang Lan. Research on the mechanism design and control mode of the food-assisting robot[D]. Harbin: Harbin Engineering University, 2007.

[10] Chen Z, Xiu D. On Generalized Residual Network for Deep Learning of Unknown Dynamical Systems[J]. Journal of Computational Physics, 2021.

[11] He K, Zhang X, Ren S, et al. Deep Residual Learning for Image Recognition[J]. IEEE, 2016.

[12] Feng X Y, Mei W, Hu D S. Aerial Target Detection Based on Improved Faster R- CNN[J]. Acta Optica Sinica, 2018, 38(6):0615004. 